\title{
Utilização do ABCDE no atendimento do traumatizado
}

\author{
Use of $A B C D E$ in caring for the traumatized
}

\section{Mateus de Sousa Rodrigues ${ }^{1}$, Leonardo Fernandes e Santana ${ }^{2}$, Ivan Martins Galvão ${ }^{3}$}

Rodrigues MS, Santana LF, Galvão IM. Utilização do ABCDE no atendimento do traumatizado / Use of ABCDE in caring for the traumatized. Rev Med (São Paulo). 2017 out.-dez.;96(4):278-80.

RESUMO: O ABCDE é um mnemônico que padroniza o atendimento inicial ao politraumatizado e define prioridades na abordagem ao trauma. Entretanto, com a aplicação prática dos protocolos, essa sistematização passa por modificações. Esse estudo sistematiza as principais condutas pré-hospitalares e hospitalares que lograram êxito no atendimento ao traumatizado. Para tanto, foram analisadas publicações recentes de revistas nacionais e internacionais. Este trabalho observou que a obstrução da via aérea é a causa de mortalidade mais rápida no politraumatizado, sendo seguida por distúrbios na ventilação e respiração e hemorragia. Por isso, a avaliação das vias aéreas com controle cervical deve ser a primeira conduta na abordagem do trauma. Com essa sistematização definida pelo ABCDE, é possível direcionar o atendimento ao politraumatizado e, com efeito, reduzir mortalidade.

Descritores: Cuidados de suporte avançado de vida no trauma/ normas; Centros de traumatologia/normas; Ferimentos e lesões.; Procedimentos clínicos/normas.

\begin{abstract}
The ABCDE is a mnemonic that standardizes initial care for the traumatized and defines an priorities in the approach to trauma. However, with a practical application of the protocols, this systematization undergoes modifications. This study systematize the main prehospital and hospital conduits that were successful in the assessment to the traumatized. For this, recent publications of national and international journals were analyzed. This study observed that an obstruction of the airway is faster cause of faster mortality in the polytraumatized, followed by disturbances in ventilation and breathing and hemorrhage. Therefore, an evaluation of the airways with cervical control should be the first step in the approach to trauma. With this systematization defined by the $\mathrm{ABCDE}$, it is possible to direct care to polytraumatized and, therefore, reduce mortality.
\end{abstract}

Keywords: Advanced trauma life support care/standards; Trauma centers/standards; Wounds and injuries; Critical pathways/standards.

1. Universidade Federal do Vale do São Francisco (UNIVASF), Petrolina, PE, BR. Discente de medicina -UNIVASF; diretor científico da Liga Acadêmica de Medicina de Urgência e Emergência (LAMURGEM-UNIVASF). Email: mateuserem@gmail.com.

2. Universidade Federal do Vale do São Francisco (UNIVASF), Petrolina, PE, BR. .Discente de medicina - UNIVASF. Email: lleonardofernandes94@gmail.com.

3. Universidade Federal do Vale do São Francisco (UNIVASF), Petrolina, PE, BR. Médico docente do curso de medicina - UNIVASF. Email: ivan.galvão@univasf.edu.br.

Endereço para correspondência: Mateus de Sousa Rodrigues. Av. José de Sá Maniçoba, s/n. Centro, Petrolina, PE. CEP: 56304-917. 


\section{INTRODUÇÃO}

ABCDE do Advanced Trauma Life Support
(ATLS) foi elaborado pelo Colégio Americano de Cirurgiões no sentido de padronizar o atendimento ao politraumatizado. Ele foi pensado para identificar lesões potencialmente fatais ao indivíduo ${ }^{1}$. Ele é aplicável a todos as vítimas com quadro crítico, independentemente da idade ${ }^{2}$.

O mnemônico ABCDE foi padronizado de acordo com as lesões de maior mortalidade. O seu significado é: A (airways) - vias aéreas com controle da coluna cervical; B (breathing) - respiração e ventilação; C (circulation) - circulação com controle da hemorragia; D (disability) estado neurológico; E (exposure) - exposição e controle da temperatura ${ }^{1}$. O ABCDE é aplicado no exame primário do atendimento inicial ao politraumatizado e é utilizado para detectar lesões de risco iminente de morte. Ele também pode ser retomado no exame secundário durante a monitorização dos sinais vitais ${ }^{3}$.

O objetivo desse trabalho é realizar uma revisão da abordagem do ABCDE no atendimento inicial ao paciente traumatizado e suas medidas auxiliares. Esse trabalho sistematiza as principais prioridades bem sucedidas no atendimento ao politraumatizado.

\section{MÉTODO}

Esta é uma revisão da importância e do significado do ABCDE no atendimento ao traumatizado. Esse trabalho sistematiza condutas pré-hospitalares e intra-hospitalares no atendimento ao traumatizado a partir de publicações recentes de revistas nacionais e internacionais. As principais publicações utilizadas nessa investigação foram: estudos prospectivos, estudos transversais e revisões sistemáticas. A consulta das revistas utilizadas por esse estudo se deu mediante o Scholar Google. Os termos utilizados durante a pesquisa nessa base de dados foram: "ABCDE AND trauma AND importance", "airways AND trauma", "breathing AND trauma", "circulation AND trauma", "disability AND trauma", "exposure AND trauma", "ABCDE do trauma".

\section{Importância e significado do ABCDE}

No A, realiza-se a proteção da coluna cervical. Em vítimas conscientes, a equipe de socorro deve se aproximar da vítima pela frente, para evitar que mova a cabeça para os lados durante o olhar, podendo causar lesões medulares ${ }^{4}$. Um membro da equipe deve estabilizar a coluna cervical manualmente ou com um colar. Se o pescoço estiver em uma posição viciosa, deve-se imobilizar nessa posição. Em vítimas inconscientes em que não é possível reposicionar a cabeça, a estabilização deve ser feita na posição original ${ }^{5}$. A imobilização deve ser de toda a coluna, não se limitando a coluna cervical. Para isso, uma prancha rígida deve ser utilizada. Os acidentes nos seguintes cenários têm riscos potenciais de lesão de medula: mergulho em água, queda de cavalo e acidentes de trânsito ${ }^{4}$.

Além disso, ainda no A, deve-se realizar a avaliação das vias aéreas. No atendimento pré-hospitalar, $66-85 \%$ das mortes evitáveis ocorrem por obstrução de vias aéreas ${ }^{6,7}$. O socorrista aborda a vítima perguntando seu nome. Se ele responder, isso sugere que as vias aéreas estão pérvias ${ }^{2,5}$. Voz alterada, estridor, roncos e esforço respiratório são sinais de obstrução de vias aéreas. Baixo nível de consciência também é uma possível causa de obstrução, geralmente pela queda da língua. As manobras mais utilizadas nessa fase são a elevação do mento (chin lift) e anteriorização da mandíbula (jaw thrust). Entretanto, é preciso ter cuidado para não causar extensão cervical nas vítimas com suspeita de lesão medular ${ }^{4}$. Quando se tem equipamentos adequados, é recomendado aspirar os corpos estranhos da via aérea ${ }^{2}$. No ambiente hospitalar, corpos estranhos de visualização direta são aspirados com fórceps de Magill ${ }^{4}$.

Quando essas medidas de desobstrução não são efetivas, três procedimentos podem ser realizados: intubação endotraqueal, cricotireoidostomia ou traqueostomia. A intubação endotraqueal é o padrão ouro para proteção de vias aéreas no ambiente pré-hospitalar. As principais indicações para este procedimento são: estresse respiratório, Escala de Coma de Glasgow menor ou igual a 8 e parada cardíaca ${ }^{2}$.

No B, o socorrista deve analisar se a respiração está adequada. A frequência respiratória, inspeção dos movimentos torácicos, cianose, desvio de traquéia e observação da musculatura acessória são parâmetros analisados nessa fase. Vítimas com hipoventilação e frequência respiratória menor que 10 inspirações por minuto devem ser monitorados ${ }^{8}$. Uma ventilação adequada deve manter as pressões parciais de $\mathrm{CO}_{2}$ entre 30-35 $\mathrm{mmHg}^{9}$. Se disponíveis, estetoscópio e o oxímetro de pulso são equipamentos que ajudam na abordagem a vítima nesse estágio. Uma vez inadequada à respiração, deve-se prestar suporte ventilatório. Nos casos de pneumotórax hipertensivo, o procedimento a ser realizado é a descompressão imediata do tórax, inserindo um jelco calibroso no segundo espaço intercostal na linha hemiclavicular, seguido de drenagem de tórax no quarto espaço intercostal, na linha axilar média ${ }^{2}$.

No $\mathrm{C}$, a circulação e a pesquisa por hemorragia são os principais parâmetros de análise. A maioria das hemorragias é estancada pela compressão direta do foco ${ }^{10}$. Quando essa medida não é suficiente, o torniquete é uma opção ${ }^{11,12}$. A frequência de pulso e enchimento capilar podem ser determinados. Mudanças na coloração da pele, sudorese e diminuição do estado de consciência podem sugerir perfusão comprometida. A ausculta pode 
Rodrigues MS, et al. Utilização do ABCDE no atendimento do traumatizado.

ser realizada nessa fase. Deve-se ter cuidado com a hipovolemia ${ }^{2}$. Nesses casos, a medida recomendada é obter dois acessos venosos periféricos e infundir, inicialmente, 2 L de cristalóides ${ }^{1}$. Entretanto, deve-se ter cautela com a diluição dos fatores de coagulação devido a esse procedimento. Em casos de fratura de pelve, dispositivos pneumáticos podem ser utilizados ${ }^{10}$. Esses equipamentos devem ser inflados $60-80 \mathrm{mmHg}$, comprimindo abdome, pelve e membros inferiores ${ }^{8}$.

No D, a análise do nível de consciência, tamanho e reatividade das pupilas, presença de hérnia cerebral, sinais de lateralização e o nível de lesão medular são medidas realizadas ${ }^{1,10}$. Nessa fase, o objetivo principal é minimizar as chances de lesão secundária pela manutenção da perfusão adequada do tecido cerebral $^{13}$. Existem duas formas de verificar o nível de consciência: o AVPU e a Escala de Coma de Glasgow (ECG). No AVPU, tem-se: A - alerta; V - responsivo à voz; $\mathrm{P}$ - responsivo à dor e $\mathrm{U}-$ irresponsivo $^{2}$. A ECG avalia a resposta motora (1-6), resposta verbal (1-5), e a abertura ocular (1-4). Nessa escala, a pontuação mínima e máxima são 3 e 15, respectivamente. ECG entre 1315, 9-12 e 3-8 sugerem normalidade, dano moderado e estado neurológico severo, respectivamente. Vítimas com Glasgow entre 3-8 precisam ser intubados. Já que queda no nível de consciência pode sugerir redução da perfusão cerebral, sendo toda queda de consciência considerada oriunda do sistema nervoso central até que o contrário

\section{REFERÊNCIAS}

1. Colégio Americano de Cirurgiões. Suporte avançado de vida no trauma para médicos. ATLS. 9a ed. Chicago; 2014.

2. Thim T, Krarup NHV, Grove EL, Rohde CV, Løfgren B. Initial assessment and treatment with the Airway, Breathing, Circulation, Disability, Exposure (ABCDE) approach. Int J Gen Med. 2012;117-21. doi: 10.2147/IJGM.S28478.

3. Pavelqueires S. MAST: manobras avançadas de suporte ao trauma. 5a ed. São Paulo: Legis Summa; 2002.

4. Beuran M, Paun S, Gaspar B, Vartic M, Hostiuc S, Chiotoroiu A, Negoi I. Prehospital trauma care: a clinical review. Chirurgia (Bucur). 2012;107:564-70. Available from: http://revistachirurgia.ro/pdfs/2012-5-564.pdf.

5. Campbell JE, Stevens JT, Charpentier L. Assessment and initial management of the trauma patient. In: JE Campbell, editor. International Trauma Life Support for Prehospital Care Providers. USA: Person Education International; 2008. p.27-44.

6. Mauger J, Deakin CD. Initial Assessment, Triage, and Basic and Advanced Life Support. In: Soreide E, Grande CM, editors. Prehospital trauma care. Boca Raton, Fl.: Taylor \& Francis; 2001. p.181-201. doi: 10.1201/b14021-13.

7. Christensen EF, Deakin CD, Vilka GM, FK Lippert. Prehospital care and trauma systems. In: Wilson WC, Grande $\mathrm{CM}$, Hoyt DB, editors. Trauma: emergency resuscitation, perioperative anesthesia and surgical management. Atlanta, seja provado. Alteração nesse nível requer reavaliação do A e B do mnemônico ${ }^{1}$. Entretanto, nesse estágio de avaliação, o abuso de drogas e a hipoglicemia devem ser $\operatorname{considerados}^{13}$. Já que também podem alterar o nível de consciência ${ }^{1}$.

No E, a análise da extensão das lesões e o controle do ambiente com prevenção da hipotermia são as principais medidas realizadas ${ }^{13}$. O socorrista deve analisar sinais de trauma, sangramento, manchas na pele etc. Além disso, deve-se despir a vítima para detectar ou excluir novas lesões. Nessa fase, deve-se medir a temperatura da vítima $^{2}$. Além disso, o ambiente de exposição (E) deve possuir uma temperatura adequada para evitar que a vítima perca calor. Depois do atendimento, a vítima deve ser coberta com cobertores aquecidos. Os cristalóides e infusões intravenosas também devem estar aquecidos ${ }^{13}$.

\section{CONCLUSÕES}

Este trabalho analisou que a obstrução da via aérea é a causa de mortalidade mais rápida no politraumatizado, sendo seguida por distúrbios na ventilação e respiração e hemorragia. Por isso, a avaliação das vias aéreas com controle cervical deve ser a primeira conduta na abordagem do trauma. Com essa sistematização definida pelo ABCDE, é possível direcionar o atendimento ao politraumatizado e, com efeito, reduzir mortalidade.

GA, Informa Health Care/Taylor \& Francis Group; 2007. p.43-58. doi:10.1001/jama.299.5.577.

8. Stratton SJ, Eckstein M. Prehospital trauma care. In: Demetriades D, Asensio JA, editors. Trauma management. Georgetown, Texas: Landes Bioscience; 2000. p.1-14.

9. Peitzman AB, Sarani B. Phase 0: Damage control resuscitation in the emergency department and pre-hospital Settings. New York: Springer Science; 2010. p.101-21. doi: 10.1007/978-0-387-89508-6_8.

10. Beuran M, Negoi I, Paun S, Runcanu A, Gaspar B, Vartic M. Trauma scores: a review of the literature. Chirurgia (Bucur). 2012;107(3):291-7.

11. Salomone JP, Solomon JHA. Prehospital care. In: Feliciano DV, Mattox KL, Moore EE, editors. Trauma. New York: McGraw Hill; 2008. p.121-39.

12. Wedmore I. Prehospital and enroute care. In: Martin M, Beekley A, editors. Front line surgery: a practical approach. New York: Springer Science + Business Media; 2011. p.116.

13. Dupanovic M. Encyclopedia of trauma care. New York: Springer; 2015. p.1,5-6

Artigo recebido em: 25.11.16

Artigo aceito em: 02.08.17 